Article

\title{
Focus of Ongoing Onchocerciasis Transmission Close to Bangui, Central African Republic
}

Eric de Smet ${ }^{1}$, Salvatore Metanmo ${ }^{2}$, Pascal Mbelesso ${ }^{3}$, Benoit Kemata ${ }^{4}$, Joseph N. Siewe Fodjo 1,5®D, Farid Boumédiène ${ }^{2}$, Hilda T. Ekwoge ${ }^{5}$, Emmanuel Yangatimbi ${ }^{3}$, Daniel Ajzenberg ${ }^{2}$, Orphee Badibanga ${ }^{6}$, Pierre-Marie Preux ${ }^{2}$ and Robert Colebunders ${ }^{1, *(1)}$

1 Global Health Institute, University of Antwerp, 2610 Antwerp, Belgium; eric.desmet@zol.be (E.d.S.); JosephNelson.SieweFodjo@uantwerpen.be (J.N.S.F.)

2 INSERM, University of Limoges, CHU Limoges, IRD, U1094 Tropical Neuroepidemiology, Institute of Epidemiology and Tropical Neurology, GEIST, 87000 Limoges, France; salvatore.metanmo@gmail.com (S.M.); farid.boumediene@unilim.fr (F.B.); ajz@unilim.fr (D.A.); pierre-marie.preux@unilim.fr (P.-M.P.)

3 Faculté des Sciences de la Santé, Université de Bangui, Bangui BP:3183, Central African Republic; pmbelesso@yahoo.com (P.M.); yang_emma1@yahoo.fr (E.Y.)

4 Onchocerciasis Control Programme, Neglected Disease Control Programme, Bangui P.O. Box 883, Central African Republic; bkemata@yahoo.fr

$5 \quad$ HILPharma Organization, Yaoundé P.O. Box 25625, Cameroon; hildaekwoge@gmail.com

6 Association to Promote Neurosciences (APRONES), Kinshasa XI P.O. Box 127,

Democratic Republic of the Congo; orpheebadibanga@gmail.com

* Correspondence: robert.colebunders@uantwerpen.be; Tel.: +32-486920149; Fax: +32-3-265-26-63

Received: 25 March 2020; Accepted: 29 April 2020; Published: 30 April 2020

\begin{abstract}
Recently, there were anecdotal reports of a high number of persons with epilepsy, including children with nodding seizures in the Landja Mboko area located about $9 \mathrm{~km}$ from the capital city Bangui, Central African Republic. We suspected the area to be endemic for onchocerciasis, and that the alleged increase in the number of epilepsy cases was due to ongoing Onchocerca volvulus transmission. However, ivermectin mass drug distribution (MDA) had never been implemented in the area. Therefore we performed an Ov16 antibody prevalence study among children, aged 6-9 years, using the biplex rapid diagnostic test (SD Bioline Oncho/LF biplex IgG4 RDT). The overall Ov16 seroprevalence was $8.9 \%$, and that of lymphatic filariasis (LF) was $1.9 \%$. Ov16 seropositivity was highest in Kodjo (20.0\%), a village close to rapids on the river. Our study shows that there is ongoing O. volvulus transmission in the Landja Mboko area. We recommend that the extent of this onchocerciasis focus should be mapped, and the introduction of ivermectin MDA should be considered in these communities.
\end{abstract}

Keywords: onchocerciasis; epilepsy; Ov16 seroprevalence; children; Central African Republic

\section{Introduction}

Onchocerciasis, a filarial disease caused by Onchocerca volvulus, is a country-wide public health problem in the Central African Republic (CAR). A rapid assessment in 2006 revealed that onchocerciasis transmission was ongoing in most parts of the country, with about 1,601,059 persons residing in meso- or hyper-endemic areas [1]. Due to the armed conflict that started in 2013, the CAR is currently confronted with a serious humanitarian crisis. As a consequence, onchocerciasis elimination efforts have been interrupted in certain parts of the country. In 2018, the estimated number of individuals requiring ivermectin treatment was 2,688,483 but it was reported that only 921,480 persons were treated with ivermectin [2]. More recent findings by CAR's Ministry of Health show that in 2019, 
the onchocerciasis endemicity mapping was not very different from the baseline assessment of 2006 (Figure 1).

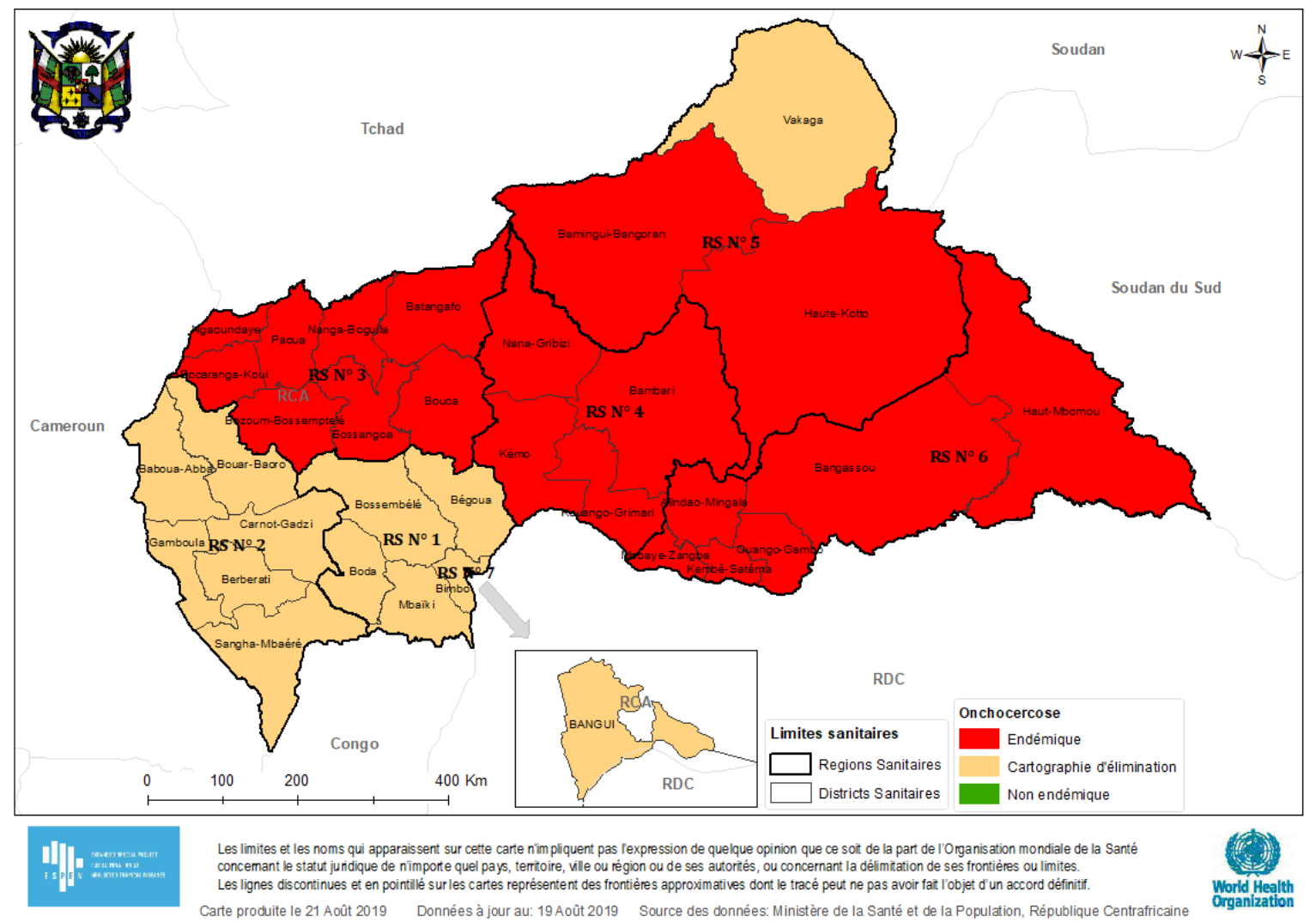

Figure 1. Onchocerciasis endemicity map of the Central African Republic, 2019.

Based on the initial rapid epidemiological mapping of onchocerciasis (REMO) studies in CAR, only the north-western areas of the country were identified as being meso- or hyper-endemic for onchocerciasis and community-directed treatment with ivermectin (CDTI) was implemented in those areas in 1993. In 1994, a population-based survey of blindness and visual impairment was conducted in the district of Bossangoa (in the Ouham river valley) [3], which showed substantial onchocerciasis-related ocular problems in the area. Besides ocular manifestations, it was also suspected that onchocerciasis may be responsible for epilepsy in the Bossangoa area. A matched case-control study was therefore conducted in 1996, which found that 39.6\% of the epileptics and $35.8 \%$ of the controls were infected with $O$. volvulus; no significant association was found between onchocerciasis and epilepsy (odds ratio $=1.21,95 \%$ confidence interval $0.81-1.80$ ) [4]. This lack of association may have been caused by previous ivermectin use in these populations, as demonstrated by previous studies [5,6]. The CAR is also considered to be endemic for lymphatic filariasis (LF) but there are no recent data about the prevalence of LF in the different parts of the country [7].

Recent anecdotal findings from CAR suggest a high burden of epilepsy, including nodding seizures, in the Landja Mboko area located about $9 \mathrm{~km}$ from the capital city Bangui. Although this area had previously been classified as being hypo-endemic for onchocerciasis and hence never benefitted from CDTI, we suspected that the increase in the number of epilepsy cases, particularly among children, was due to ongoing $O$. volvulus transmission. We therefore conducted community-based surveys to confirm this anecdotal epilepsy burden and also assess the onchocerciasis situation in 2020. This paper reports our preliminary findings regarding the current onchocerciasis transmission status in the villages of interest. 


\section{Methods}

The study was conducted in January 2020 in the villages of Landja Mboko, in the south western part of CAR, about $9 \mathrm{~km}$ from the capital city Bangui (Figure 2).

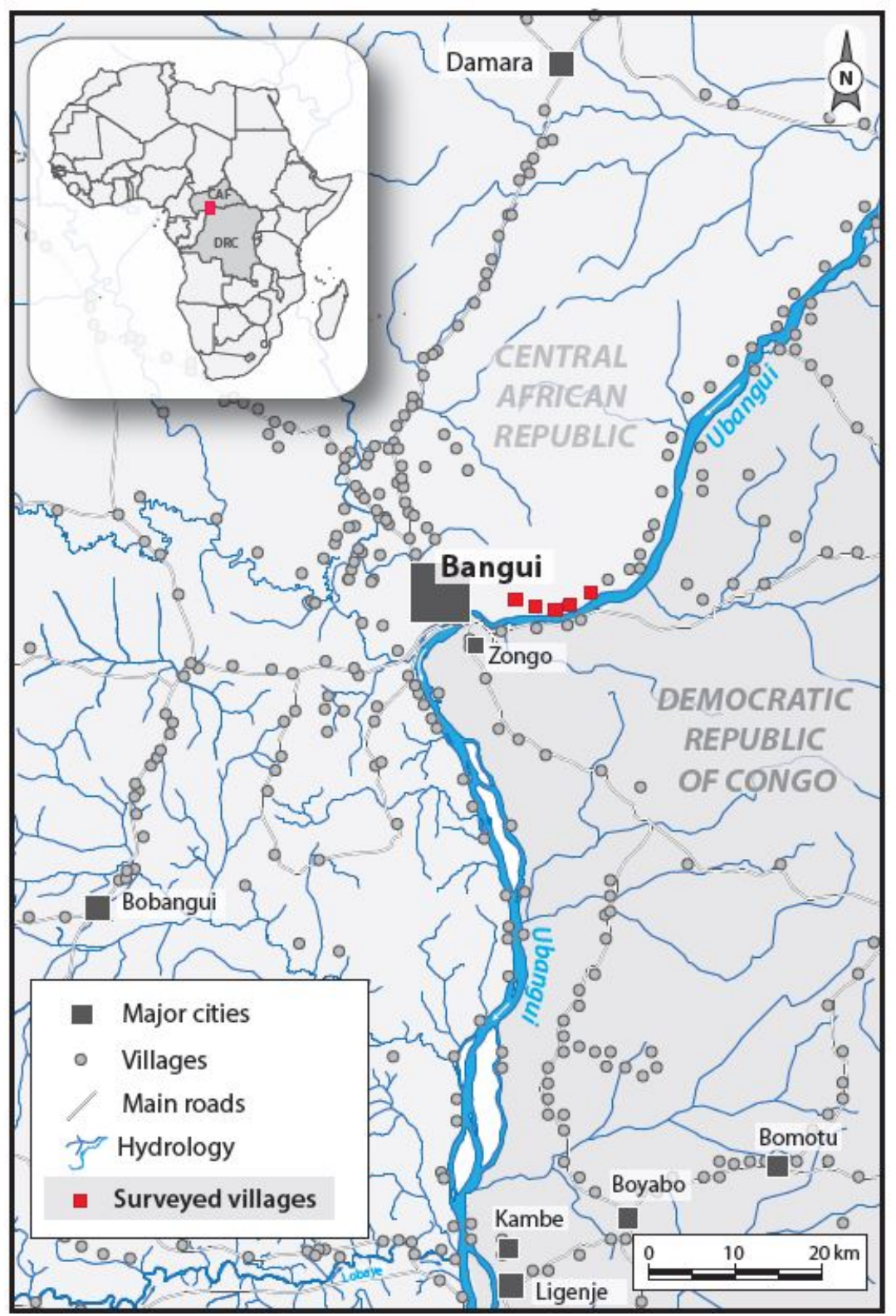

Figure 2. The villages of Landja Mboko included in the study.

Using a door-to-door approach, non-epileptic children aged 6-9 years of both sexes were recruited for an Ov16 seroprevalence study; the 5-9 years age group is indeed recommended by the World Health Organization (WHO) when assessing ongoing onchocerciasis transmission patterns [8]. We excluded children suffering from any known illness or for whom we did not obtain assent/parental consent to participate. The socio-demographic and anthropometric information of participants was noted, and they were all tested for Ov16 IgG4 antibodies using a biplex rapid diagnostic test (SD Bioline Oncho/LF biplex IgG4 RDT), which also measures exposure to lymphatic filariasis (LF). Using rigorous aseptic procedures, the participants were finger-pricked to obtain a drop of blood; the tests were performed as per the manufacturer's instructions, and the results were noted for each participant. 


\section{Results}

A total of 259 children aged 6-9 years from five villages were included in this study (Table 1); 135 (52.1\%) of them were males. Participants in Kodjo and Landja 2 villages had significantly higher median ages (7.5 years), compared to the other study villages where the median age was 7.0 years (Kruskal Wallis $p$-value $=0.025)$. Only two $(0.8 \%)$ participants reported ever taking ivermectin in the past, because they happened to be in CDTI areas during the ivermectin distribution period.

Table 1. Characteristics of study participants.

\begin{tabular}{|c|c|c|c|c|c|}
\hline & $\begin{array}{c}\text { Belespoir } \\
\mathrm{n}=30\end{array}$ & $\begin{array}{l}\text { Kodjo } \\
\mathrm{n}=50\end{array}$ & $\begin{array}{c}\text { Landja } 1 \\
\mathrm{n}=49\end{array}$ & $\begin{array}{c}\text { Landja } 2 \\
n=106\end{array}$ & $\begin{array}{c}\text { Mangapou } 2 \\
n=24\end{array}$ \\
\hline \multicolumn{6}{|c|}{ Socio-demographics } \\
\hline Male: n (\%) & $19(63.3)$ & $25(50.0)$ & $20(40.8)$ & $58(54.7)$ & $13(54.2)$ \\
\hline Female: $\mathrm{n}(\%)$ & $11(36.7)$ & $25(50.0)$ & $29(59.2)$ & $48(45.3)$ & $11(45.8)$ \\
\hline Age: median (IQR) & $7.0(6.0-7.0)$ & $7.5(6.0-8.8)$ & $7.0(6.0-8.0)$ & $7.5(6.0-9.0)$ & $7.0(6.0-8.0)$ \\
\hline $\begin{array}{l}\text { Duration in village: } \\
\text { median (IQR) }\end{array}$ & $7.0(6.0-7.0)$ & $7.0(6.0-8.0)$ & $7.0(6.0-8.0)$ & $7.0(6.0-8.0)$ & $7.0(6.0-8.0)$ \\
\hline Enrolled in school: $\mathrm{n}(\%)$ & $30(100.0)$ & $43(86.0)$ & $43(87.8)$ & $90(84.9)$ & $20(83.3)$ \\
\hline School level: median (IQR) & $1(1-2)$ & $2(1-3)$ & $1(1-2)$ & $2(1-2)$ & $2(1-2)$ \\
\hline \multicolumn{6}{|c|}{ Anthropometrics } \\
\hline Height in cm: median (IQR) & $113(110-121)$ & $112(108-119)$ & $114(110-118)$ & $119(112-125)$ & $112(105-120)$ \\
\hline HfA $^{\text {a }}$ z-score: mean (SD) & $-1.04(1.27)$ & $-1.81(1.51)$ & $-1.12(1.00)$ & $-1.00(1.38)$ & $-2.25(2.29)$ \\
\hline Stunting $^{\mathrm{b}}: \mathrm{n}(\%)$ & $6(20.0)$ & $22(44.0)$ & $8(16.7)$ & $27(25.7)$ & $13(54.2)$ \\
\hline Weight in kg: median (IQR) & $20.5(20.0-24.8)$ & $20.0(15.2-23.0)$ & $22.0(20.0-25.0)$ & $22.0(19.0-25.0)$ & $20.5(16.8-24.2)$ \\
\hline BfA $^{\mathrm{c}} \mathrm{z}$-score: mean (SD) & $0.71(0.80)$ & $-0.67(2.97)$ & $0.60(1.74)$ & $-0.23(1.71)$ & $0.83(2.06)$ \\
\hline Underweight $^{\mathrm{d}}$ : $\mathrm{n}(\%)$ & $0(0)$ & $16(32.0)$ & $3(6.3)$ & $15(14.4)$ & $1(4.2)$ \\
\hline
\end{tabular}

${ }^{a}$ HfA: Height-for-age z-score based on the WHO growth curves; ${ }^{b}$ Stunting: Height-for-age $<-2 z .{ }^{c}$ BfA: BMI-for-age $\mathrm{z}$-score based on the WHO growth curves; ${ }^{d}$ Underweight: BMI-for-age $<-2 z$. IQR: Interquartile range; SD: Standard deviation.

The overall Ov16 seroprevalence was 8.9\%, and that of LF was 1.9\% (Table 2). Ov16 seropositivity varied significantly across the villages, with the highest values in Kodjo village (20.0\%), followed by Landja $1(14.3 \%)$, Landja 2 (4.7\%), Mangapou 2 (4.2\%), and Belespoir (0\%).

Table 2. Results of the rapid diagnostic tests.

\begin{tabular}{cccccc}
\hline & Belespoir & Kodjo & Landja 1 & Landja 2 & Mangapou 2 \\
\hline \multicolumn{5}{c}{ Age-specific Ov16 results: $\mathbf{n}$ (\%) } \\
\hline 6 years & $0(0)$ & $2(10.0)$ & $4(17.4)$ & $1(2.8)$ & $0(0)$ \\
\hline 7 years & $0(0)$ & $2(40.0)$ & $1(7.7)$ & $0(0)$ & $0(0)$ \\
\hline 8 years & $0(0)$ & $4(33.3)$ & $1(11.1)$ & $3(13.6)$ & $1(14.3)$ \\
\hline 9 years & $0(0)$ & $2(15.4)$ & $1(25.0)$ & $1(3.2)$ & $0(0)$ \\
\hline Overall $^{\text {a }}$ & $0(0)$ & $10(20.0)$ & $7(14.3)$ & $5(4.7)$ & $1(4.2)$ \\
\hline & Age-specific Lymphatic Filariasis (LF) results: $\mathbf{n}(\%)$ & \\
\hline 6 years & $0(0)$ & $0(0)$ & $0(0)$ & $1(2.8)$ & $0(0)$ \\
\hline 7 years & $0(0)$ & $0(0)$ & $0(0)$ & $0(0)$ & $0(0)$ \\
\hline 8 years & $0(0)$ & $0(0)$ & $0(0)$ & $2(9.5)$ & $0(0)$ \\
\hline 9 years & $0(0)$ & $1(7.7)$ & $0(0)$ & $1(3.2)$ & $0(0)$ \\
\hline Overall $^{\mathrm{b}}$ & $0(0)$ & $1(2.0)$ & $0(0)$ & $4(3.8)$ & $0(0)$ \\
\hline
\end{tabular}

a Overall Ov16 seroprevalence: $23 / 259$ (8.9\%); Difference across villages (Fisher exact test): $p=0.004 .{ }^{\mathrm{b}}$ Overall LF seroprevalence: 5/258 (1.9\%); Difference across villages (Fisher exact test): $p=0.739$. 
The median age of Ov16-positive participants was higher than that of Ov16-negative children, although the difference was not significant ( 8 years vs. 7 years; Mann-Whitney $U$-value $=0.378$ ). However, more Ov16-positive children were underweight than their Ov16-negative counterparts; $p=$ 0.018 (Table 3).

Table 3. Comparison of Ov16-positive and Ov16-negative participants.

\begin{tabular}{lccc}
\hline & $\begin{array}{c}\text { Ov16 Negative } \\
\mathbf{n = 2 3 6}\end{array}$ & $\begin{array}{c}\text { Ov16 Positive } \\
\mathbf{n = 2 3}\end{array}$ & $p$-Value \\
\hline Age: median (IQR) & $7.0(6.0-8.0)$ & $8.0(6.0-8.0)$ & $0.378^{\mathrm{a}}$ \\
\hline Male gender: $\mathrm{n}(\%)$ & $125(53.0)$ & $10(43.5)$ & $0.515^{\mathrm{b}}$ \\
\hline School level: median (IQR) & $2(1-2)$ & $2(1-3)$ & $0.569^{\mathrm{a}}$ \\
\hline Stunting: $\mathrm{n}(\%)$ & $67(28.5)$ & $9(40.9)$ & $0.330^{\mathrm{b}}$ \\
\hline Underweight: $\mathrm{n}(\%)$ & $28(12.0)$ & $7(31.8)$ & $0.018^{\mathrm{b}}$ \\
\hline Itching: $\mathrm{n}(\%)$ & $83(35.9)$ & $14(60.9)$ & $0.034^{\mathrm{b}}$ \\
\hline LF seropositivity: $\mathrm{n}(\%)$ & $3(1.3)$ & $2(9.1)$ & $0.059^{\mathrm{c}}$ \\
\hline${ }^{\mathrm{b}}$ Mann-Whitney U test; ${ }^{\mathrm{b}}$ Chi-squared test; ${ }^{\mathrm{c}}$ Fisher exact test. LF: Lymphatic filariasis. IQR: Interquartile range.
\end{tabular}

\section{Discussion}

Our results show that there is ongoing onchocerciasis and LF transmission in the Landja Mboko area of CAR. The Ov16 seropositivity rate of $8.9 \%$ in children < 10 years is far above the $1 \%$ threshold proposed by the WHO in deciding whether an area should be benefitting from mass treatment with ivermectin [8]. Given that this region was also mapped as being loiasis-endemic [9], care must be taken when deciding which onchocerciasis elimination strategies are to be implemented in such a setting to avoid severe adverse events due to ivermectin treatment [10]. Hence, alternative strategies such as test-and-not-treat and vector control approaches could be considered [8,11].

Particular attention must be given to Kodjo village, where children had a much higher Ov16 seroprevalence than in all other study sites. Compared to other villages, Kodjo is situated only $200 \mathrm{~m}$ away from river Oubangui which most likely constitutes a suitable breeding ground for blackflies (the vector that transmits the onchocerciasis parasite). Previous research has documented an increasing burden of not only onchocerciasis, but also epilepsy, as the distance from the breeding ground decreases [12]. A similar phenomenon may explain the anecdotal reports of frequent epilepsy in this area. The findings from the door-to-door epilepsy survey in this same area will be published separately and may indeed shed more light on the current situation in Landja Mboko.

In our study, we observed that Ov16-positive children reported more frequent itching and were more often underweight compared to those who tested negative. A similar trend was observed when assessing the frequency of stunting in both groups, albeit being statistically non-significant. While retarded growth has previously been associated with onchocerciasis [13], it is unclear from this study whether the low anthropometric parameters observed in our participants were due to nutritional problems or exposure to O. volvulus.

A limitation of this study is the fact that only Ov16 rapid tests were used to assess onchocerciasis transmission, without confirmation using ELISA (the gold standard technique for Ov16 antibody detection, more sensitive than rapid tests) nor complementing our research with entomology. Our study area is also considered to be endemic for loiasis [9], but we did not determine the prevalence of Loa loa infection in our study population. While LF detection using RDT may suffer from cross-reactions with Loa loa [14], the performance of the Ov16 IgG rapid test in detecting exposure to O. volvulus is very satisfactory: sensitivity of $89.1 \%(95 \%$ CI: $86.2 \%-92.0 \%)$ and specificity of $97 \%(95 \%$ CI: 95.4\%-98.6\%) [15]. Our results therefore provide compelling evidence that Landja Mboko constitutes a hitherto ignored onchocerciasis focus in the CAR, requiring urgent attention from the public health authorities. 


\section{Conclusions}

Our data shows that there is ongoing O. volvulus transmission in the Landja Mboko area, where there is co-endemicity with loiasis. We also noted the occurrence of LF, although to a much lesser extent than onchocerciasis. Therefore a more in-depth re-assessment of the onchocerciasis situation (via skin snip surveys and entomological studies) in this area is warranted. Given the risk of encephalopathy when introducing ivermectin mass drug administration in loiasis-endemic areas, the prevalence and intensity of $L$. loa infection also needs to be assessed in these villages. In the light of the paradigm shift from control to elimination of onchocerciasis, it is crucial that all transmission foci be identified and appropriate interventions initiated.

Author Contributions: Conceptualization: E.d.S., J.N.S.F., R.C. and D.A.; Methodology: R.C. and J.N.S.F.; Investigation: E.d.S.; P.M., E.Y., S.M., B.K., O.B.; Data entry and cleaning: H.T.E.; Formal Analysis: J.N.S.F.; Writing-Original Draft Preparation R.C.; Writing—review and editing: all authors; Visualization F.B., Supervision R.C. and P.M., Project Administration, R.C. and D.A., Funding acquisition: R.C. and P.-M.P. All authors have read and agreed to the published version of the manuscript.

Funding: This research was funded by the European Research Council, grant number 671055 (R.C.).

Acknowledgments: We thank the local health personnel and community workers.

Conflicts of Interest: The authors declare no conflicts of interest.

\section{References}

1. World Health Organization. African Programme for Onchocerciasis Control (APOC) | Central African Republic. 2006. Available online: https://www.who.int/apoc/countries/caf/en/ (accessed on 1 March 2020).

2. World Health Organization. Reported Number of Individuals Treated for Onchocerciasis: Data by Country. Global Health Observatory Data Repository. 2018. Available online: http://apps.who.int/gho/data/node. main.NTDONCTREAT?lang=en (accessed on 1 March 2020).

3. Schwartz, E.C.; Huss, R.; Hopkins, A.; Dadjim, B.; Madjitoloum, P.; Henault, C.; Klauss, V. Blindness and visual impairment in a region endemic for onchocerciasis in the Central African Republic. Br. J. Ophthalmol. 1997, 81, 443-447. [CrossRef] [PubMed]

4. Druet-Cabanac, M.; Preux, P.-M.; Bouteille, B.; Bernet-Bernady, P.; Dunand, J.; Hopkins, A.; Yaya, G.; Tabo, A.; Sartoris, C.; Macharia, W.; et al. Onchocerciasis and Epilepsy: A Matched Case-Control Study in the Central African Republic. Am. J. Epidemiol. 1999, 149, 565-570. [CrossRef] [PubMed]

5. Levick, B.; Laudisoit, A.; Tepage, F.; Ensoy-Musoro, C.; Mandro, M.; Bonareri Osoro, C.; Suykerbuyk, P.; Kashama, J.M.; Komba, M.; Tagoto, A.; et al. High prevalence of epilepsy in onchocerciasis endemic regions in the Democratic Republic of the Congo. PLoS Negl. Trop. Dis. 2017, 11, e005732. [CrossRef] [PubMed]

6. Colebunders, R.; Mandro, M.; Mokili, J.L.; Mucinya, G.; Mambandu, G.; Pfarr, K.; Reiter-Owona, I.; Hoerauf, A.; Tepage, F.; Levick, B.; et al. Risk factors for epilepsy in Bas-Uélé Province, Democratic Republic of the Congo: A case-control study. Int. J. Infect. Dis. 2016, 49, 1-8. [CrossRef] [PubMed]

7. World Health Organization. Global Programme to Eliminate Lymphatic Filariasis: Progress Report, 2017; World Health Organization: Geneva, Switzerland, 2018. Available online: http://apps.who.int/iris/bitstream/handle/ 10665/275719/WER9344.pdf?ua=1 (accessed on 30 April 2020).

8. World Health Organization. Report of the Second Meeting of the WHO Onchocerciasis Technical Advisory Subgroup; World Health Organization: Geneva, Switzerland, 2018. Available online: https://apps.who.int/iris/bitstream/ handle/10665/277238/WHO-CDS-NTD-PCT-2018.11-eng.pdf?ua=1 (accessed on 30 April 2020).

9. Zouré, H.G.M.; Wanji, S.; Noma, M.; Amazigo, U.V.; Diggle, P.J.; Tekle, A.H.; Remme, J.H.F. The Geographic Distribution of Loa loa in Africa: Results of Large-Scale Implementation of the Rapid Assessment Procedure for Loiasis (RAPLOA). PLoS Negl. Trop. Dis. 2011, 5, e1210. [CrossRef] [PubMed]

10. Boussinesq, M.; Gardon, J.; Gardon-Wendel, N.; Chippaux, J.-P. Clinical picture, epidemiology and outcome of Loa-associated serious adverse events related to mass ivermectin treatment of onchocerciasis in Cameroon. Filaria J. 2003, 2, S4. [CrossRef] [PubMed]

11. Boussinesq, M.; Fobi, G.; Kuesel, A.C. Alternative treatment strategies to accelerate the elimination of onchocerciasis. Int. Health 2018, 10, i40-i48. [CrossRef] [PubMed] 
12. Boussinesq, M.; Pion, S.D.; Ngangue, D.; Kamgno, J. Relationship between onchocerciasis and epilepsy: A matched case-control study in the Mbam Valley, Republic of Cameroon. Trans. R. Soc. Trop. Med. Hyg. 2002, 96, 537-541. [CrossRef]

13. Kipp, W.; Bamuhiiga, J.; Burnham, G.; Leichsenring, M. The Nakalanga Syndrome in Kabarole District, Western Uganda. Am. J. Trop. Med. Hyg. 1996, 54, 80-83. [CrossRef]

14. Pion, S.D.; Montavon, C.; Chesnais, C.B.; Kamgno, J.; Wanji, S.; Klion, A.D.; Nutman, T.B.; Boussinesq, M. Positivity of Antigen Tests Used for Diagnosis of Lymphatic Filariasis in Individuals Without Wuchereria bancrofti Infection But with High Loa loa Microfilaremia. Am. J. Trop. Med. Hyg. 2016, 95, 1417-1423. [CrossRef]

15. Golden, A.; Steel, C.; Yokobe, L.; Jackson, E.; Barney, R.; Kubofcik, J.; Peck, R.; Unnasch, T.R.; Nutman, T.B.; de los Santos, T.; et al. Extended Result Reading Window in Lateral Flow Tests Detecting Exposure to Onchocerca volvulus: A New Technology to Improve Epidemiological Surveillance Tools. PLoS ONE 2013, 8, e69231. [CrossRef] [PubMed]

(C) 2020 by the authors. Licensee MDPI, Basel, Switzerland. This article is an open access article distributed under the terms and conditions of the Creative Commons Attribution (CC BY) license (http://creativecommons.org/licenses/by/4.0/). 